\title{
Stress-free vesicle production
}

\section{ce \\ by modulating \\ the export \\ of POS it is \\ possible to \\ regulate the \\ production of \\ OMVs}

Outer-membrane vesicles (OMVs) are spherical structures that bud from the bacterial outer membrane and are most commonly produced by Gramnegative bacteria. Their composition and organization resemble that of the outer membrane and they are involved in several functions, including the promotion of pathogenesis, bacterial survival during stress and the regulation of microbial interactions within bacterial communities. Despite their importance, little is known about their biogenesis.

A recent study by Florez et al. investigated the mechanisms that underlie the biogenesis of OMVs in Pseudomonas aeruginosa in the absence of external membrane stresses that are already known to influence this process. The authors found that the export of a quorum sensing molecule known as Pseudomonas quinolone signal (PQS) promoted their biogenesis. According to a previous model that was proposed by the same group, the incorporation of PQS into the outer

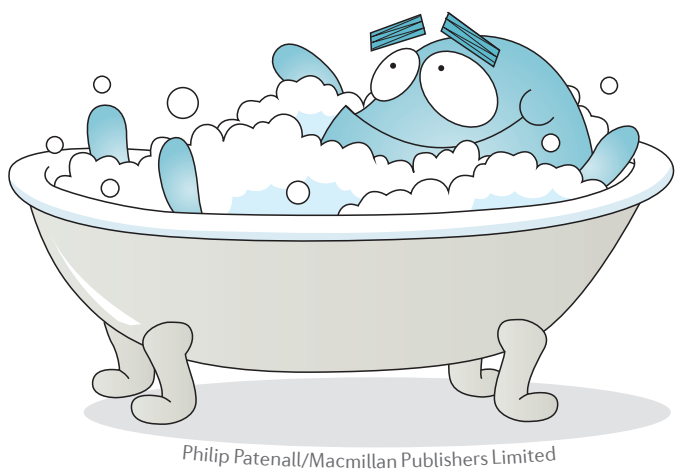

leaflet of the outer membrane causes an expansion of the membrane that induces bacterial membrane curvature and the subsequent release of OMVs. Therefore, the authors hypothesized that PQS had to be transported from the cytoplasm to the outer membrane to initiate the biogenesis of OMVs.

To test this hypothesis and confirm that the export of PQS was necessary for OMV biogenesis, they first analysed the production and export of PQS, and the production of OMVs, in two laboratory strains of $P$. aeruginosa. This analysis revealed substantial strain differences both in the amount of OMVs that were produced and in the amount of PQS that was released into the culture supernatant. In particular, the POA1 strain that was shown to produce less OMVs had a larger relative proportion of intracellular PQS compared with the PA14 strain that produced more OMVs and released a greater amount of PQS into the extracellular space. Despite these differences, both strains showed comparable growth properties and produced similar amounts of PQS, which suggests that the spatial distribution of PQS determined the amount of OMVs that were produced.

Indeed, by separating the bacterial inner and outer membranes, and quantifying the amount of PQS in the different fractions, they observed that in the POA1 strain that did not export PQS and did not secrete
OMVs efficiently, the majority of PQS was confined to the inner membrane, whereas only a small percentage of PQS was found in the inner membrane of strain PA14. Moreover, both strains were shown to have a point of saturation at which the export of PQS decreased and intracellular accumulation started, and strain POA1 was shown to be saturated at a much lower extracellular concentration than strain PA14.

In addition, the authors found that by growing the two strains in the richer brain-heart infusion medium, rather than in Luria-Bertani medium, they could increase the export of PQS and the subsequent production of OMVs in both strains, which suggests that nutrient and ion availability have an important role in PQS secretion and could be manipulated to alter the production of OMVs. Finally, the authors tested three distinct clinical isolates and confirmed the results obtained in laboratory strains, highlighting the relevance of this mechanism for bacterial physiology and pathogenesis. This study shows a direct correlation between the export of PQS and OMV biogenesis, which suggests that by modulating the export of PQS it is possible to regulate the production of OMVs.

Irene Vacca

ORIGINAL ARTICLE Florez, C. et al. Membrane distribution of the Pseudomonas quinolone signal modulates outer membrane vesicle production in Pseudomonas aeruginosae. mBio 8, e01034-17 (2017) 